\title{
Microscopic theory of spatially resolved photoluminescence in quantum structures
}

\author{
G Pistone, S Savasta, O Di Stefano and R Girlanda \\ Istituto Nazionale per la Fisica della Materia INFM and Dipartimento di Fisica della \\ Materia e Tecnologie Fisiche Avanzate, Università di Messina, Salita Sperone 31, \\ I-98166 Messina, Italy \\ E-mail: pistone@unime.it
}

\begin{abstract}
We present a microscopic quantum theory of spatially resolved photoluminescence in quantum wells with interface fluctuations. The theory can model low-temperature photoluminescence and photoluminescence excitation experiments performed in illumination, collection, illumination-collection mode or diffusion experiments where the spatial positions of excitation and collection are scanned independently. Numerically calculated two-dimensional images clarify the impact of the microscope setup on the obtained images and resolutions.
\end{abstract}

\section{Introduction}

In the last few years measurements based on spatiallyresolved photoluminescence (PL) provided direct information on the spatial and energy distribution of light emitting nanometric dots of semiconductor quantum structures, thus opening a rich area of physics involving spatially resolved quantum systems [1-5]. Detailed simulations of Zimmermann et al have clarified many aspects of the intriguing nonequilibrium dynamics giving rise to photoluminescence spectra in disordered quantum structures [6, 7]. However, theoretical simulations of near-field imaging spectroscopy of semiconductor quantum structures focus on calculations of local absorption [8-11]. In contrast, as a matter of fact, almost all experimental images are obtained from PL measurements. Here we present a microscopic quantum theory of spatially resolved photoluminescence in quantum structures, which includes both light quantization (essential to describe spontaneous emission) and phonon scattering. The theory includes the description of spatially confined excitation (illumination mode) and/or detection (collection mode). This formulation thus permits us to model spectroscopic imaging based on PL excitation spectroscopy in which the excitation and detection energies and spatial positions can all be scanned independently.

\section{Theory}

The positive frequency components of the operator describing the signal that can be detected by a general near-field setup can be expressed as [12] $\hat{S}_{t}^{+}=\hat{A}_{\mathrm{bg}}^{+}+\hat{S}^{+}$, where $\hat{A}_{\mathrm{bg}}^{+}$is the elastic background signal (largely uniform along the $x-y$-plane) proportional to the input electric-field operator and $\hat{S}^{+}=$ $\mathcal{A} \int \mathrm{d} \mathbf{r} \hat{\mathbf{P}}^{+}(\mathbf{r}) \cdot \mathbf{E}_{\text {out }}(\mathbf{r})$ is related to the sample polarization density operator $\hat{\mathbf{P}}^{+}(\mathbf{r})$, where $\mathcal{A}$ is a complex constant depending on the impedance of the material constituting the tip [12] and $\mathbf{E}_{\text {out }}(\mathbf{r})$ is the signal mode delivered by the tip. The interband polarization density operator can be expressed in terms of exciton operators as $\hat{\mathbf{P}}^{+}(\mathbf{r})=$ $\boldsymbol{\mu}_{e h} f(z) \Psi_{\alpha}^{e h}(\rho=0, \mathbf{R}) \hat{B}_{\alpha}$. The operator $\hat{B}_{\alpha}^{\dagger}$ creates an exciton state (one electron-hole pair) $\hat{B}_{\alpha}^{\dagger}|0\rangle \equiv\left|E_{1, \alpha}\right\rangle$ with energy $\omega_{1, \alpha}$. $\Psi_{\alpha}^{e h}(\rho, \mathbf{R})$ is the exciton wavefunction with $\rho$ indicating the relative in-plane $e h$ coordinate and $\mathbf{R}$ describing the centre of mass motion, while $f(z)=u_{e}(z) u_{h}(z)$ is the product of the electron and hole envelope functions along the confinement direction (the growth axis). If the disorder induced broadening is small compared to the exciton binding energy, only the lowest bound state $1 \mathrm{~s}$ at the fundamental sublevel transition has to be considered and the exciton wavefunction can be factorized as [7] $\Psi_{\alpha}^{e h}(\rho, \mathbf{R})=\phi_{1 s}(\rho) \psi_{\alpha}(\mathbf{R})$. The steady-state spectrum of incoherent light emitted by the semiconductor quantum structure and detected by the near-field setup can be expressed as

$$
I_{\mathrm{PL}}\left(\omega_{\text {out }}\right)=\frac{1}{\pi} \int_{0}^{\infty} \mathrm{d} \tau\left\langle\hat{S}^{-}(0) \hat{S}^{+}(\tau)\right\rangle \mathrm{e}^{\mathrm{i} \omega_{d} \tau}
$$

The kinetic equation for diagonal terms of the exciton density matrix $N_{\alpha}=\left\langle\hat{B}_{\alpha}^{\dagger} \hat{B}_{\alpha}\right\rangle$ can be derived starting from the Heisenberg equation of motion under the influence of a 

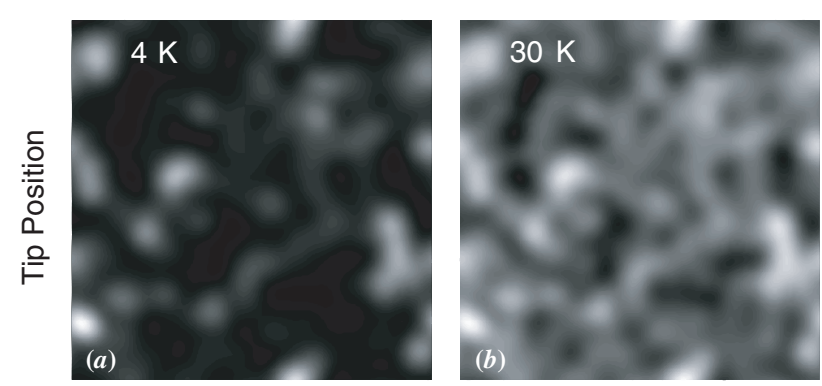

Tip Position

Figure 1. (a) PL spectrum when the sample has a temperature of $T=4 \mathrm{~K}$, (b) PL spectrum when the sample has a temperature of $T=30 \mathrm{~K}$.

Hamiltonian describing the interaction with acoustic phonons and with a (possibly inhomogeneous) light field. Assuming a low-density regime, one obtains

$$
\partial_{t} N_{\alpha}=G_{\alpha}\left(\omega_{\text {in }}\right)+\sum_{\beta} \gamma_{\alpha \leftarrow \beta} N_{\beta}-2 \Gamma_{\alpha} N_{\alpha}
$$

where $\gamma_{\beta \leftarrow \alpha}$ are the resulting phonon-assisted scattering rates [7]. $2 \Gamma_{\alpha}=r_{\alpha}+\sum_{\beta} \gamma_{\beta \leftarrow \alpha}$ is the total out-scattering rate, $r_{\alpha}$ being the rate for spontaneous emission proportional to the exciton oscillator strength: $r_{\alpha}=r_{0}\left|\int \mathrm{d}^{2} \mathbf{R} \psi_{\alpha}(\mathbf{R})\right|^{2}$. In this equation the generation term depends on the spatial overlap between the illuminating beam and the exciton wavefunctions corresponding to exciton levels resonant with the input light and can thus be a function of the tip position and shape: $G_{\alpha}=r_{0}\left|o_{\alpha}^{\text {in }}\right|^{2} \mathcal{L}_{\alpha}\left(\omega_{\text {in }}\right)$ with $\pi \mathcal{L}_{\alpha}(\omega)=\Gamma /\left[\left(\omega-\omega_{\alpha}\right)^{2}+\Gamma^{2}\right]$ and $o_{\alpha}^{\text {in }}=\int \mathrm{d}^{2} \mathbf{R} \tilde{E}_{\text {in }}(\mathbf{R}) \psi_{\alpha}(\mathbf{R})$ where $\tilde{E}_{\text {in }}(\mathbf{R})=\int E_{\text {in }}(\mathbf{r}) f(z) \mathrm{d} z$. In numerical calculations concerning the illumination mode we will assume an input light field with a given Gaussian profile centred around the tip position: $\tilde{E}_{\text {in }}(\mathbf{R})=E_{\text {in }}^{0} g(\mathbf{R}-\overline{\mathbf{R}})$. In this case the generation term becomes a function of the beam position and shape (spatial resolution). We observe that also at steady state equation (2) can give rise to highly non-equilibrium exciton densities. Non-equilibrium here arises from both spontaneous emission that prevents full thermalization and from the possible local excitation described by the generation terms. Once the exciton densities have been derived, the PL spectrum can be readily obtained. According to the quantum regression theorem, $\left\langle\hat{S}^{-}(0) \hat{S}^{+}(\tau)\right\rangle$ has the same dynamics as $\left\langle\hat{S}^{+}(\tau)\right\rangle$ (proportional to the exciton operator), but with $\left\langle\hat{S}^{-}(0) \hat{S}^{+}(0)\right\rangle$ as initial condition. Following this procedure we obtain

$$
I_{\mathrm{PL}}\left(\omega_{\text {out }}\right)=r_{0} \sum_{\alpha}\left|o_{\alpha}^{\text {out }}\right|^{2} \mathcal{L}_{\alpha}\left(\omega_{\text {out }}\right) N_{\alpha}
$$

\section{Numerical results}

As a first check of the above-developed theoretical scheme we present some numerical results for spatially resolved PL. We consider a system of QDs arising from interface fluctuations of GaAs quantum wells. The effective disordered potential $V(\mathbf{r})$, used in our simulations, is obtained by summing up two different contributions. They are both modelled as a zero mean, Gauss distributed and spatially correlated process with

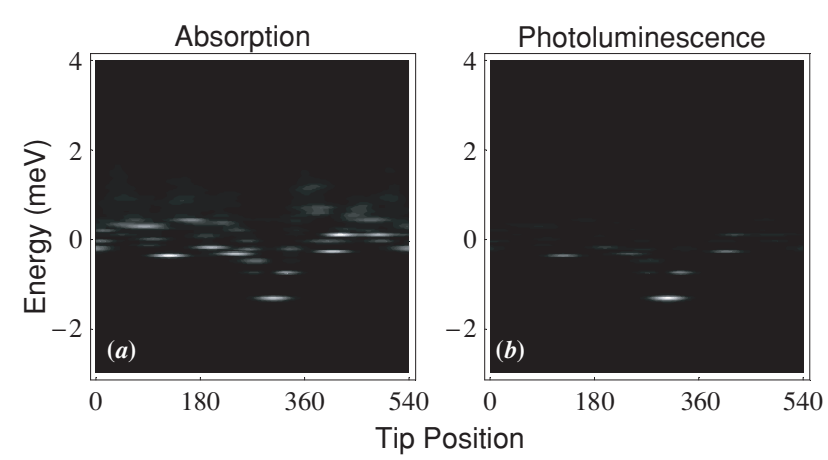

Figure 2. Line scan spectra obtained as a function of the beam position and energy $I_{\mathrm{PL}}\left(X_{0}, Y, \omega\right)$ with $X_{0}$ fixed: $(a)$ absorption spectrum; $(b)$ photoluminescence spectrum. The temperature of the sample is $T=4 \mathrm{~K}$. Spatial resolution of the collecting tip: $\mathrm{FWHM}=47 \mathrm{~nm}$.

prescribed amplitude $v_{0}$ and correlation length $\xi$. For the two contributions we have chosen $\xi=16 \mathrm{~nm}, v_{0}=1.5 \mathrm{meV}$ and $\xi=8 \mathrm{~nm}$ and $v_{0}=0.5 \mathrm{meV}$ respectively. Figure 1 displays two energy-integrated PL images $I(X, Y)$ obtained at two different temperatures ( $T=4$ and $30 \mathrm{~K}$ respectively). These spectra are obtained after uniform illumination of the sample at energy $\omega=1 \mathrm{meV}$ and collecting locally the emitted light (collection mode with spatial resolution FWHM = $47 \mathrm{~nm}$ ). When passing from $T=30 \mathrm{~K}$ to $T=4 \mathrm{~K}$, we observe the transition from a broad and fairly continuous emission to an intense set of spatially localized emission peaks in correspondence with the potential minima. This behaviour is also observed in numerous experiments. It can be instructive to better understand the emission mechanisms of these systems to compare measurements of spatially resolved absorption spectra (recently an indirect method able to provide information on near-field absorption has been developed [14]) with corresponding PL spectra. Figure 2 displays absorption and the corresponding photoluminescence line-scan spectra calculated at $T=4 \mathrm{~K}$ for the sample. These line scans are a function of the beam position and energy $I_{\mathrm{PL}}\left(X_{0}, Y, \omega\right)$ with $X_{0}$ fixed. We observe how in the PL spectrum (figure 2(b)) evidence of states at higher energy due to the continuous band coming from quasi-2D states originating from the potential barriers, present in the absorption spectrum (figure 2(a)), disappears. This originates from the fact that for these states energy relaxation due to phonon scattering is much more rapid than the radiative decay rate.

\section{References}

[1] Hess H F et al 1994 Science 2641740

[2] Gammon D et al 1995 Appl. Phys. Lett. 672391

[3] Gammon D et al 1996 Phys. Rev. Lett. 763005 Gammon D et al 1996 Science 27387

[4] Wu Q et al 1999 Phys. Rev. Lett. 832652

[5] Hasen J et al 1997 Nature 39054

[6] Zimmermann R and Runge E 1997 Phys. Status Solidi a 164511

[7] Zimmermann R et al 2003 Theory of resonant secondary emission: Rayleigh scattering versus luminescence Quantum Coherence, Correlation and Decoherence in 
Semiconductor Nanostructures ed T Takagahara (Amsterdam: Elsevier) pp 89-165

[8] Mauritz O et al 1999 Phys. Rev. Lett. 82847

[9] Di Stefano O et al 2000 Appl. Phys. Lett. 772804

[10] Di Stefano O et al 2002 J. Appl. Phys. 912302
[11] Pistone G et al 2003 Phys. Rev. B 67153305

[12] Greffet J-J and Carminati R 1997 Prog. Surf. Sci. 56 133

[13] Axt V M et al 1996 Phys. Rev. B 537244

[14] Guest J R et al 2001 Science 2932224 